\title{
Distinct Developmental Modes and Lesion-Induced Reactions of Dendrites of Two Classes of Drosophila Sensory Neurons
}

\author{
Kaoru Sugimura, ${ }^{1,2}$ Misato Yamamoto, ${ }^{1,3}$ Ryusuke Niwa, ${ }^{1,5}$ Daisuke Satoh, ${ }^{1,2}$ Satoshi Goto, ${ }^{4}$ Misako Taniguchi, ${ }^{4}$ \\ Shigeo Hayashi, ${ }^{4}$ and Tadashi Uemura ${ }^{1,5}$ \\ ${ }^{1}$ Department of Molecular Genetics, The Institute for Virus Research, ${ }^{2}$ Graduate School of Science, and ${ }^{3}$ Graduate School of Biostudies, Kyoto University, \\ Kyoto 606-8507, Japan, ${ }^{4}$ The Genetic Stock Research Center at the National Institute of Genetics for Drosophila Stocks, National Institute of Genetics, \\ Mishima, Shizuoka 411-8540, Japan, and ${ }^{5}$ Core Research for Evolutional Science and Technology, Japan Science and Technology, Kawaguchi 332-0012, \\ Japan
}

Little has been understood about the underlying mechanisms that generate the morphological diversity of dendritic trees. Dendritic arborization neurons in Drosophila provide an excellent model system to tackle this question, and they are classified into classes I-IV in order of increasing arbor complexity. Here we have developed transgenic green fluorescent protein markers for class I or class IV cells, which allowed time-lapse recordings of dendritic birth in the embryo, its maturation processes in the larva, and lesion-induced reactions. The two classes used distinct strategies of dendritic emergence from the cell body and branching, which contributed to differences in their basic arbor patterns. In contrast to the class I cells examined, one cell of class IV, which was a focus in this study, continued to elaborate branches throughout larval stages, and it was much more capable of responding to the severing of branches. We also investigated the cellular basis of field formation between adjacent class IV cells. Our results support the fact that class-specific inhibitory interaction is necessary and sufficient for tiling and confirmed that this intercellular communication was at work at individual dendrodendritic interfaces. Finally, this inhibitory signaling appeared to play a central role when arbors of adjacent cells started meeting midway between the cells and until the body wall became partitioned into abutting, minimal-overlapping territories.

Key words: dendrites; Drosophila; time-lapse analysis; GAL4 enhancer trap; laser ablation; tiling

\section{Introduction}

Terminal morphologies of dendritic trees are not only complex, but they are also variable to a considerable extent from one neuronal type to another, probably manifesting a unique physiological role of each type (Ramón y Cajal, 1995; MacNeil and Masland, 1998; Elston, 2000). One of important questions about dendritic pattern formation is how individual types of neurons control branching and achieve distinct complexities. Another question centers around how the dendritic territory of each type is shaped. For example, in the vertebrate retina, a receptive field belonging to a cell of one particular type of ganglion or amacrine

Received Nov. 4, 2002; revised Feb. 13, 2003; accepted Feb. 18, 2003.

This work was supported by grants from the Ministry of Science, Culture and Education to T.U. and S.G.; a grant from Toray Foundation (Japan) for the Promotion of Science to T.U.; a grant from Core Research for Evolutional Science and Technology to T.U.; and a grant from the Japan Society for the Promotion of Science to S.H. R.N. was the recipient of a Fellowship of the Japan Society for the Promotion of Science for Junior Scientists. We are grateful to Eric Wieschaus, Trudi Schüpbach, Akinao Nose, the Berkeley Drosophila Genome Project, and the Bloomington Stock Center for Drosophila stocks, and Kenji Nagai and Akira Miyawaki for the Venus plasmids. We also thank Hiroko Takeuchi and Yuko Yamaguchi for their work at initial phases, Masatoshi Takeichi for allowing us to start this project in his laboratory, and Wesley B. Grueber and Yuh Nung Jan for discussing unpublished data.

Correspondence should be addressed to Tadashi Uemura, Department of Molecular Genetics, The Institute for Virus Research, Kyoto University, Syogo-in, Kawahara-cho 53, Sakyo-ku, Kyoto 606-8507, Japan. E-mail: tuemura@virus.kyoto-u.ac.jp.

R. Niwa's present address: Department of Integrated Biosciences, Room 201, Graduate School of Frontier Sciences, The University of Tokyo, Chiba 277-8562, Japan.

S. Goto's present address: Mitsubishi Kagaku Institute of Life Sciences, Machida, Tokyo 194-8511, Japan.

S. Hayashi's present address: Riken Center for Developmental Biology, Chuo-ku, Kobe, Hyogo 650-0047, Japan. Copyright $\odot 2003$ Society for Neuroscience $\quad$ 0270-6474/03/233752-09\$15.00/0 cells overlaps only a little with other cells of the same type, and no parts of the retina fail to be covered by a receptive field of that cell type (Wässle et al., 1981; Devries and Baylor, 1997; MacNeil and Masland, 1998). This complete but nonredundant innervation of a field is referred to as "tiling."

The Drosophila peripheral nervous system (PNS) organizes a stereotyped pattern of identified sensory neurons (Bodmer and Jan, 1987; Jan and Jan, 1993; Campos-Ortega and Hartenstein, 1997). Sensory neurons include cells with multiple dendritic (MD) projections, i.e., MD neurons, and one major subtype of MD neurons is the dendritic arborization (DA) neuron that extends highly branched dendrites underneath the epidermis (see Fig. $1 \mathrm{~A}$ ). At least one subset of DA neurons may sense mechanical stimuli on the basis of analogy to the roles of morphologically similar cells in Manduca (Grueber et al., 2001). In contrast to stratified dendrites of densely packed neurons in other systems in Drosophila and other animal species, dendrites of sparsely distributed DA neurons grow and branch within two dimensions, which makes analysis of panoramas straightforward. Dendritic trees of a single or adjacent DA neurons were recently imaged in wholemount third-instar larvae (Grueber et al., 2002). This study shows that 15 DA neurons in each hemisegment A2-A6 can be classified into four categories, classes I-IV, in order of increasing arbor complexity, and that tiling occurs between class III and class IV cells. Because these data were collected from mature larva, but not from younger ones, how branching complexities of individual class cells are controlled and how the dendritic exclu- 


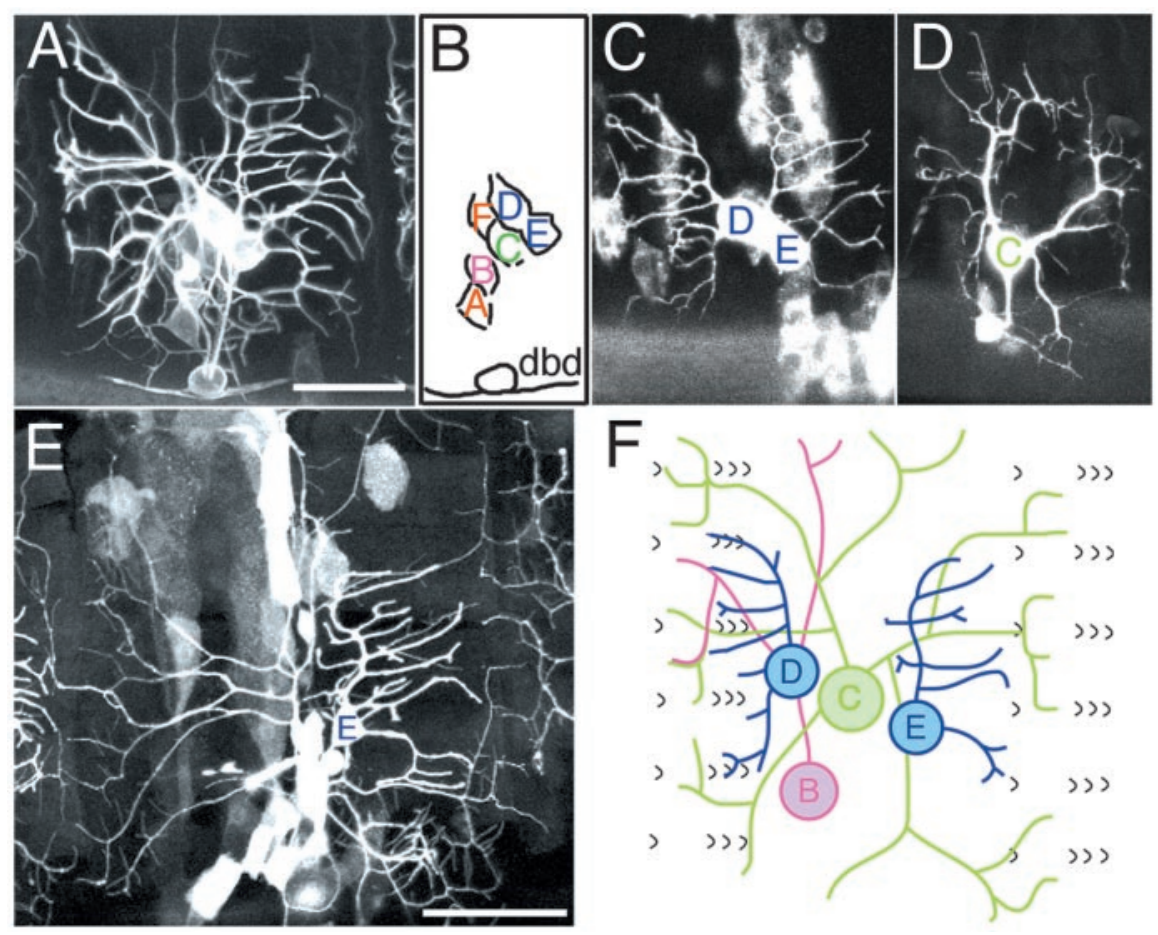

Figure 1. Markers for DA neurons. $A-D$, Multiple dendrite neurons in the dorsal cluster in the Drosophila embryo just before hatching. In this and all subsequent figures, anterior is to the left and dorsal is at the top. Data of other clusters are not shown. $A$, $B, A$ pan da marker. An image of Venus-pm $/+; \mid G 1-2 /+(A)$ and schematic representation of cell bodies of ddaA-ddaF and dorsal bipolar dendrite $(\mathrm{dbd})$ neuron $(B)$. C, ddaD and ddaE are highlighted in NP2225/mCD8::GFP. D, Among DA neurons in the dorsal cluster, only ddaC is reproducibly visualized in NP7028 GFP[S65T]/GFP[S65T]. E, Larva of 45.5- $47.5 \mathrm{hr}$ after egg laying (AEL). ddaE is strongly labeled in homozygotes of IG1-1 GFP[S65T] mCD8::GFP. F, A diagram of the dorsal cluster in a hemisegment, showing a spatial relationship between dendritic arbors of ddaB-E and epidermal denticles (black symbols). For simplicity, cell bodies are illustrated as if they were not clustered. Branches of $d d a D$ and those of $d d a E$ reach the anterior three rows and the most posterior row of denticles, respectively. The space between the most posterior row of a hemisegment and the most anterior one in a posterior neighbor is referred to the neutral zone, where ddaB and C are innervated. Scale bars: $A-D, 20 \mu \mathrm{m} ; E, 50 \mu \mathrm{m}$. were visible in 61 strains, and 4 of the 61 were further characterized and included in this study.

DA marker strains, green fluorescent protein reporters, and other Drosophila strains. The four strains examined in this study were IG1-1, IG1-2, NP2225, and NP7028; all of them have pGawB insertions on the second chromosome. "NP" stands for strains that were established by the NP consortium (Hayashi et al., 2002) (http://flymap.lab.nig.ac.jp/ dclust/getdb.html), and IG1-1 and IG1-2 were derived from the collection of Schüpbach and Wieschaus (1998). GAL4 expression in IG1-2 decreased after hatching. UAS transgenes of green fluorescent protein (GFP) variants frequently used were GFP[S65T] (Bloomington stock center \#1521), mCD8::GFP (Lee and Luo, 1999), GFP-actin (Verkhusha et al., 1999), tauGFP (Brand, 1995), Venus, and Venus-pm (described next). Generated recombinant chromosomes of GAL4 insertions and one or two GFP transgenes were IG1-1 GFP[S65T] mCD8::GFP, IG1-2 GFP[S65T], NP7028 GFP[S65T], and NP7028 mCD8::GFP. Using a given GAL4 line, expression of membranebound reporter proteins ( $\mathrm{mCD} 8:$ GFP and Venus-pm) and that of unfused forms (GFP[S65T] and Venus) gave essentially similar patterns, except that terminal branches looked thicker when visualized with membrane-bound reporters. ddaD and ddaE were observed in NP2225/mCD8::GFP that gave lower epidermal fluorescence than NP2225/GFP[S65T]. GFP-actin tended to accumulate around growing tips, whereas TauGFP was distributed to all branches of ddaD and ddaE, but only to major branches of ddaC (data not shown).

Fluorescence intensity of dendrites and celltype specificity depended on copy numbers of sion between class III and class IV neurons is accomplished remains to be solved.

Toward a full understanding of how dendrites of each class acquire their distinct shape, we generated markers for class I or IV neurons and performed time-lapse imaging of dorsal dendritic arborization neuron $\mathrm{D}(\mathrm{ddaD})$ and ddaE (class I) and ddaC (class IV) neurons. The two classes were found to use distinct strategies of dendrite outgrowth and branching in development, and ddaC cells possessed a much greater capability of responding to voided regions that were generated by microsurgery. Our results of cell ablation and branch-severing experiments strongly suggest that ddaC-ddaCspecific inhibitory recognition plays a pivotal role in dendritic tiling and provide experimental evidence for this intercellular communication functioning at each dendrodendritic interface. In addition, the inhibition appeared to be crucial in tiling until the body wall became subdivided into minimal-overlapping territories.

\section{Materials and Methods}

GAL4 enhancer trap screening. Two collections of pGawB insertions (total of 4750 strains) were screened for markers for DA neurons (Schüpbach and Wieschaus, 1998; Hayashi et al., 2002). Each GAL4 line was crossed to $y w$; UAS-GFPN-lacZ (Shiga et al., 1996), UAS-GFP[S65T], and collected embryos were stained with anti- $\beta$-galactosidase antibody ( $\mathrm{Ha}-$ yashi et al., 2002). In 191 strains, all or a subset of DA neurons seemed to be labeled at stage 15-17, and each of those lines was crossed to UASGFP[S65T] or UAS-GFPN-lacZ UAS-GFP[S65T], and fluorescent cells were examined in very late embryos or in first-instar larvae. Dendrites
GAL4 or GFP, or both, and the developmental stage. An increase in the copy number of GAL4 or GFP intensified the fluorescence as expected but tended to kill cell-type specificity. For example, we observed ddaC mostly in NP7028 GFP[S65T]/GFP[S65T], but two to three additional DA neurons sometimes showed up in homozygotes of NP7028 GFP[S65T] or NP7028 mCD8::GFP. GAL4-109(2)80 GFP[S65T], a pan-DA marker (Gao et al., 1999), and MHC-CD8-GFP-Sh (Zito et al., 1999) have been described previously. All fly embryos and larvae were grown at $25^{\circ} \mathrm{C}$.

Molecular cloning. Inserted into pUAST (Brand and Perrimon, 1993) were cDNA for Venus, a yellow fluorescent protein variant that undergoes fast and efficient maturation at $37^{\circ} \mathrm{C}$ (Nagai et al., 2002), or a made. The Venus-pm construct was made by adding a sequence encoding the $21 \mathrm{~N}$-terminal amino acids of Lyn to the $\mathrm{N}$ terminal of Venus (Sawano et al., 2002; K. Nagai and A. Miyawaki, unpublished data).

Larval anesthesia and time-lapse analysis. Anesthetizing of larvae has been described briefly (Zito et al., 1999). Larval development lasts for $\sim 4$ $\mathrm{d}$ at $25^{\circ} \mathrm{C}$. A larva, which is younger than $55 \mathrm{hr}$ after egg laying (AEL), was transferred to an apple agar plate or to a drop of $50 \%$ glycerol in PBS on a plate, and the plate was placed in a glass chamber. Kimwipe, moistened with ether, was put next to the plate; and the chamber was then sealed. Larvae were anesthetized by ether fumes for 1-4 min. Cuticles became less fragile when larvae were in 50\% glycerol in PBS. Each larva was then mounted on a slide between spacers made of vinyl tapes. During imaging, the larvae were immersed in 50\% glycerol PBS under a coverslip. Thereafter, each larva was immediately placed in a drop of $50 \%$ glycerol PBS on an apple agar plate with yeast paste and kept in a moist chamber. Larvae membrane-bound form of Venus, Venus-pm, and transgenic flies were 
older than $\sim 55 \mathrm{hr}$ AEL were exposed directly to ether fumes; otherwise it was difficult to anesthetize them, although the direct exposure reduced their viability. Dechorionated embryos were mounted in halocarbon oil, and time-lapse analysis was done as described previously (Gao et al., 1999; Oda and Tsukita, 1999). For 10-15 min, time-lapse recordings of larvae, third-instar larvae were cut along one lateral side and mounted in saline on special slides with an oxygen-permeable membrane (Yellow Spring Instrument) as described (Girdham and O'Farrell, 1994). After this surgery, larvae often shrank up, and this shrinkage made observation of dendritic tiling along segment boundaries difficult.

Laser ablation and image collection and processing. In our surgery experiments, embryos and larvae were mounted as described above, and neurons were ablated or dendrites were severed by using a laser system (Micropoint, Photonics Instruments). Because embryos were not anesthetized, laser energy was adjusted so that a cell was killed quickly by several to 10 pulses at a frequency of $12 \mathrm{~Hz}$. Within $10-30 \mathrm{sec}$ after the surgery, a number of fluorescent bumps were seen on ablated cells or on detached branches of ddaC cells, and fluorescence of cell bodies was no longer detected after $1 \mathrm{~min}$. Confocal images were collected by using 488 or $514 \mathrm{~nm}$ lasers (Bio-Rad or Zeiss) and processed by using Photoshop (Adobe Systems).

\section{Results}

\section{Isolation of DA markers}

We conducted a GAL4 enhancer trap screening and searched for GAL4 insertion lines that may work as either pan-DA or a subset of DA markers. Each candidate line was crossed with a strain having UAS-GFP transgenes, and labeled cells were identified by observing patterns of individual dendrite arbors in whole-mount embryos and first-instar larvae (see details in Materials and Methods). Four DA marker strains were under study here: IG1-1, IG1-2, NP2225, and NP7028. IG1-2 drove GFP expression in all MD neurons but not in chordotonal $(\mathrm{CH})$ or external sensory neurons with monopolar dendrites in all clusters (Fig. $1 A, B$ ). Except for IG1-2, the other three strains labeled a subset of $\mathrm{CH}$ neurons as well, which sometimes made images of the ventral, ventral-prime, and lateral clusters difficult to interpret. Thus we focused mostly on the dorsal cluster in abdominal segments $2-6$ that has each class of DA neurons but no $\mathrm{CH}$ neuron. The dorsal cluster has two class I cells (ddaD and ddaE) and one class IV cell (ddaC). NP2225 and IG1-1 are class I markers. NP2225 marked ddaD, ddaE, and ventral posterior da (vpda) (a cell in the ventral cluster), and IG1-1 labeled ddaE and vpda (Fig. 1C,E). NP7028 labeled all three class IV neurons, ddaC, ventral-prime anterior $\mathrm{da}$ ( $\left.\mathrm{v}^{\prime} \mathrm{ada}\right)$, and ventral da B (vdaB) (Fig. 1D). NP2225 and NP7028 were mainly used in subsequent studies unless described otherwise.

Distinct modes of initial dendrite formation and branching of ddaD, ddaE, and ddaC in the embryo

Dendrite formation of individual DA neurons was studied by 10 min time-lapse recordings in the embryo. ddaD and ddaE neurons were found to differ from $\mathrm{ddaC}$ neurons in their ways of
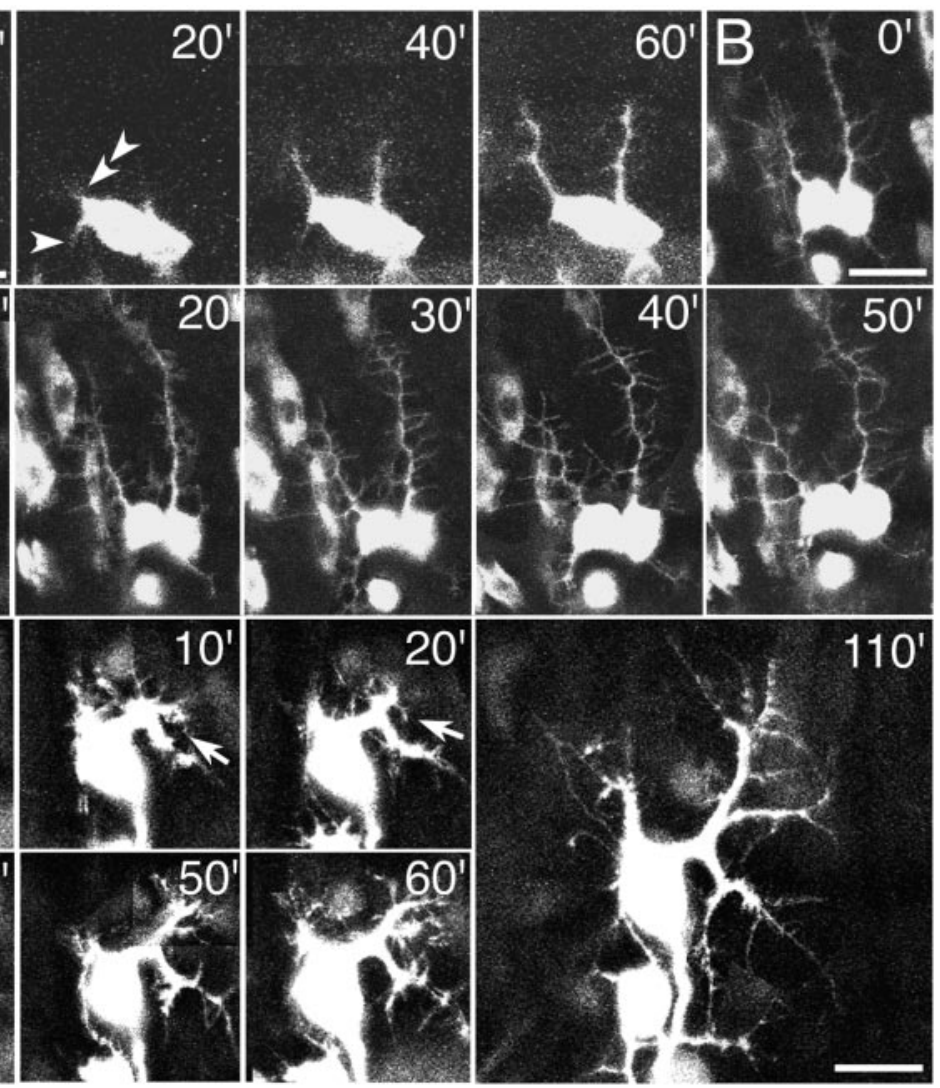

110

Figure 2. Dendritic emergence and branching in $\mathrm{ddaC}$, ddaD, and $\mathrm{ddaE}$ in the embryo. $A, B$, ddaD and $\mathrm{ddaE}$ in NP2225/mCD8::GFP. A, Images 20 min apart are shown of 10 min time-lapse recordings of an embryo at $13-14 \mathrm{hr}$ AEL. Two primary

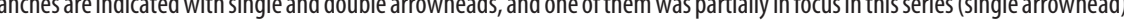
a dendritic end is indicated (arrow). Scale bar: $A-C, 10 \mu \mathrm{m}$.

initial dendrite formation and branching (Fig. 2). In both ddaD and ddaE, a first-order branch with a simple growing tip emerged at 13-14 hr AEL, which was followed by one or two additional first-order branches (Fig. $2 \mathrm{~A}$, arrowheads). Within $1 \mathrm{hr}$, a number of lateral/second-order branches sprouted from the side of the first-order branches and underwent repeated cycles of extension and retraction until a subset was stabilized by $\sim 18 \mathrm{hr}$ AEL (Fig. 2B). These observations are consistent with those reported for a pan-DA marker (Gao et al., 1999).

When cell bodies of $\mathrm{ddaC}$ were first visible at $15.5-16.5 \mathrm{hr}$ AEL, all of the neurons had already initiated dendrite formation (Fig. 2C). Every ddaC cell body was associated with two or three growing dendrite roots. In contrast to the morphologically simple tips of $\mathrm{ddaD}$ and $\mathrm{ddaE}$, ends of $\mathrm{ddaC}$ dendrites looked like growth cones with numerous filopodia. $\mathrm{ddaC}$ increased in arbor complexity by repeated bifurcation of the ends (Fig. 2C, arrows); thus it was difficult to distinguish first-order and higher-order branches. The above different styles of the two classes resulted in distinct arbor patterns.

ddaD and ddaE, but not ddaC, almost fixed the shape of overall dendritic arbors at early larval stages

After hatching $\sim 20-22 \mathrm{hr}$ AEL, dendrite development of a given DA neuron was observed in larvae that had been anesthetized with ether fumes. We traced 20 pairs of $\mathrm{ddaD}$ and ddaE by taking a series of two or three images 13-15 hr apart, beginning at 24-25 hr AEL (Fig. $3 A, B$ ). Although the two neurons continued growth 

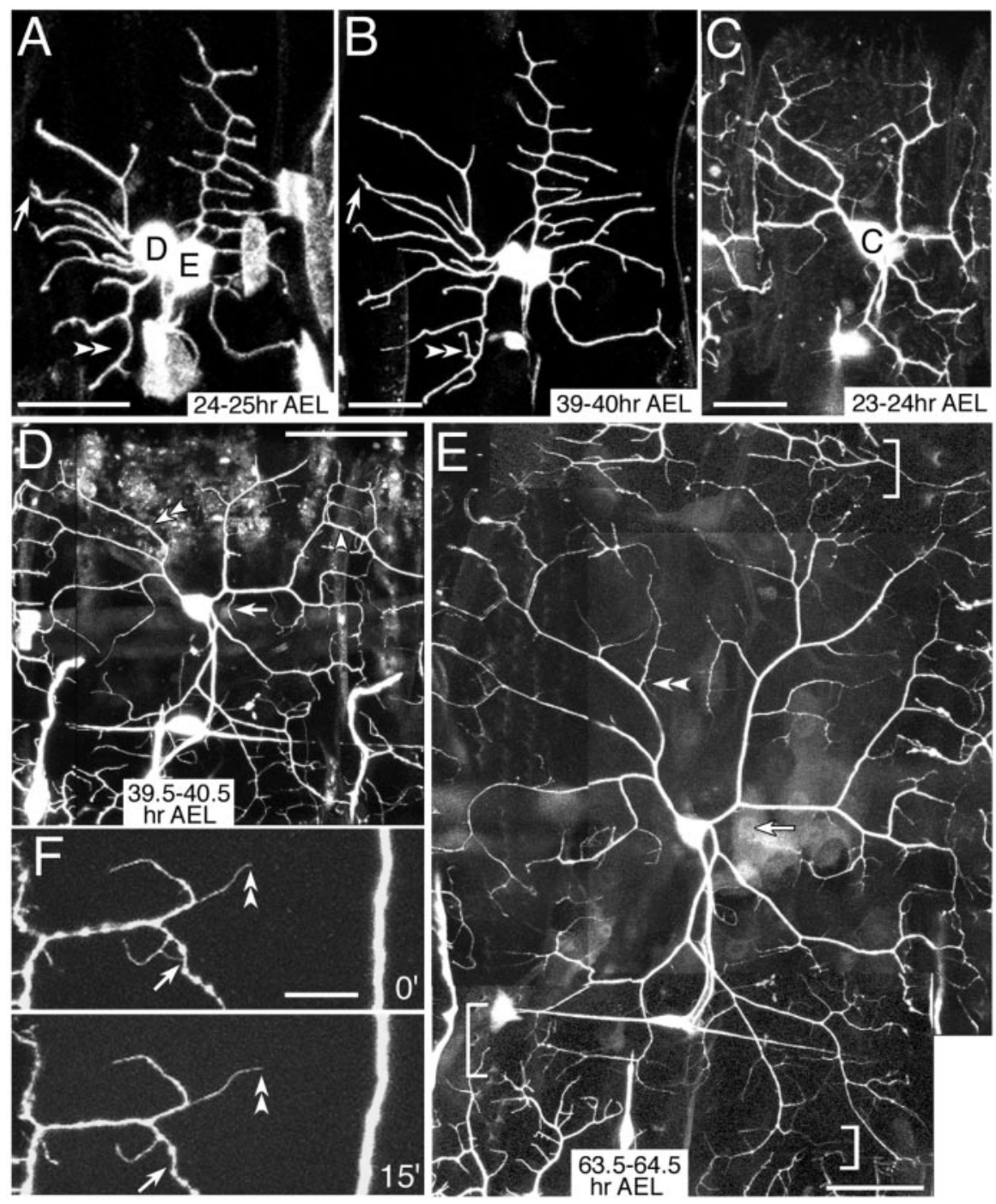

Figure 3. Formation of higher-order branches in ddaC, ddaD, and ddaE in the larva. $A, B$, ddaD and ddaE in NP2225/mCD8::GFP $(-F$, ddaC in NP7028 GFP[S65T]/GFP[S65T]. Indicated are formation or elongation of lateral branches (double arrowheads), pruning or retraction of branches (arrows), and a dendrodendritic contact (single arrowhead in D). $A, B$, Two images $15 \mathrm{hr}$ apart starting at $24-25 \mathrm{hr}$ AEL. C $-E$, Three time-lapse images taken at $23-24 \mathrm{hr}$ AEL, 39.5-40.5 hr AEL, and 63.5- $64.5 \mathrm{hr}$ AEL, respectively. $E$ Dendritic tiling between $\mathrm{dda} C$ and its contralateral homolog and between $\mathrm{dda}\left(\right.$ and $v^{\prime}$ ada in the ventral prime cluster are indicated (top bracket and bottom brackets, respectively).F, 15 min recordings of dendritic terminals in a third-instar larva. Scale bar: $A-C$, $F, 20 \mu \mathrm{m} ; D, E, 50 \mu \mathrm{m}$.

in a coordinated manner with expansion of the body surface, these cells essentially stabilized the shape of overall arbors at early larval stages. Only two to three lateral/second- or higher-order branches were generated per cell during the initial 13-15 hr of larval development (Fig. 3B, double arrowheads), and new branches were hardly seen for the next 13-15 hr. Dendrite observation from $50 \mathrm{hr}$ AEL onward was difficult in the NP2225 larva because of an increase in the number of GFP-expressing epidermal cells. Patterns of dendrite arbors of ddaE of mature thirdinstar larva were compared with those at earlier stages by using IG1-1 (Fig. 1E), and our results confirmed that ddaE achieved its final complexity possibly by $50 \mathrm{hr}$ AEL (data not shown).

Dendrite growth of a total of $33 \mathrm{ddaC}$ cells in larvae was tracked by taking a series of two or three images at $15 \mathrm{hr}$ or much longer intervals (Fig. $3 C-E$ ). In contrast to ddaD and ddaE, ddaC showed a considerable increase in the extent of branching complexity during larval stages. A subset of branches extended their terminals toward either segmental boundaries or the dorsal midline, or ventrally. Consequently those from ipsilateral or contralateral segmental homologs started filling in the open space between them, and branch terminals of $\mathrm{ddaC}$ and $\mathrm{v}^{\prime}$ ada came close to each other (details are explained later).

In addition to branch formation at or close to ends of branches, $\mathrm{ddaC}$ produced lateral fine branches far behind the ends (Fig. 3E, double arrowheads). Neither around the terminals nor in a domain more proximal to the cell body, branches of the same cell hardly overlapped. This isoneuronal (same cell) avoidance could be achieved by contact-mediated repulsion, which would be difficult to monitor by time-lapse recordings of anesthetized animals at intervals of several hours. To attain higher temporal resolution, we tracked dendritic terminals at $10-15 \mathrm{~min}$ intervals in mature larvae ( $\sim 4 \mathrm{~d}$ old $)$ that had been cut along one lateral side and mounted. This protocol captured five sets of two images 15 min apart, displaying a dendrodendritic contact and subsequent retraction of fine processes (Fig. $3 F$, arrows). Apart from retraction of the fine processes after isoneuronal contacts, we infrequently found pruning of branches in the cell types examined (Fig. $3 A, B, D, E$, arrows).

In response to severing of branches, other isoneuronal branches of $\mathrm{ddaC}$, but not those of ddaE, filled in voided fields with minimum overlap

To address whether competitive interaction occurs between growing isoneuronal branches, we severed branches by using a laser beam and examined how other branches of the same cell reacted to the voided areas. When branches of ddaC were severed at 40-43 $\mathrm{hr}$ AEL, lateral branches sprang from neighboring isoneuronal dendrites on the side of the severing and appeared to grow into voided regions that would have been supposedly covered by detached branches ( 18 of 18 cells) (Fig. $4 A, B$, bracket and single arrowheads). These newly formed branches exhibited mutual exclusion and minimum overlap with each other. This result suggests competitive interaction between isoneuronal branches of $\mathrm{ddaC}$ in normal development. In 8 of the 18 cells, we found branches that extended from the cut end (Fig. $4 A, B$, arrows), and these branches may have sprouted out from the wound.

ddaE also showed non-crossing outgrowth of isoneuronal dendrites; however, its branches did not show the type of plasticity exhibited by ddaC. This was shown by cutting branches at 20-22 hr AEL, when ddaE was still forming lateral branches $(n=$ $8)$, or at $40-42 \mathrm{hr}$ AEL when the overall shape of arbors was almost fixed $(n=6)$. The operated ddaE extended its remaining branches as far as the normal cell did; nonetheless, neighboring 
dendrites extended only short lateral branches or those that hardly sprouted laterally (Fig. 4C,D). The neighboring dendrites did not redirect their growth toward the open area either.

\section{Dendritic terminals of adjacent ddaCs} encountered each other and then kept closely apposed throughout subsequent larval stages

Terminal portions of $\mathrm{ddaC}$ branches, which extended toward segmental boundaries after hatching, formed an array at regular spaces by $35 \mathrm{hr}$ AEL (Figs. $3 D, E, 5 A, B)$. This "ladder" pattern consisted of 7-10 dendrite terminals running almost parallel along the anteroposterior axis. Dendrites from a pair of neighboring segments either interdigitated or met each other underneath the epidermis that forms the naked cuticle; thus we tentatively called the basal area of this epidermal region the "neutral" zone (Figs. $1 F$ and its legend, $5 B$, brackets, $6 A, D)$. Within the neutral zone, dendrite terminals coincided with grooves at the ends of muscle fibers (Fig. 5C,D) that were attached to the basal side of specialized epidermal cells or tendon cells (Becker et al., 1997).

Close observation of the dendritic ladder showed that terminals of dendrites from adjacent cells were closely apposed or had turned away before crossing each other (heteroneuronal avoidance) (Fig. $5 A$, arrow). Sometimes it was difficult to identify dendritic tips, and thus those tips appeared to form dendrodendritic contacts (Figs. $3 D$, arrowheads, $5 A, B$ ). Minimal overlap was also seen between dendrites extending from ddaCs in contralateral hemisegments and between ddaCs and v'ada (class IV) in the ventral prime cluster (Fig. 3E, brackets) (Gao et al., 2000; Grueber et al., 2002). By $35 \mathrm{hr}$ AEL, these dendrites met approximately midway between the two cells, as did dendrites of ipsilateral hemisegmental ddaCs, and the body wall was partitioned into abutting, nonoverlapping territories. To summarize our time-lapse recordings of $\mathrm{ddaC}$, we saw neither heteroneuronal nor isoneuronal dendrites heavily overlap or intertwine initially and then withdraw, suggesting that the dendritic avoidance occurs throughout larval development.

\section{Ablation of ddaC caused dendrite in-growth of adjacent ddaCs, but that of other DA neurons did not}

To examine putative roles of recognition between adjacent ddaCs, we ablated ddaC in one segment in the embryo and observed how it affected the bounds of dendritic fields. Even in the absence of ddaC, dendrite terminals of ipsilateral adjacent homologs navigated across the neutral zones at regular spaces on both sides of the operated hemisegments (all of 14 ablations) (Fig. $6 \mathrm{~A}$ ). Some of those dendrites transgressed bounds of the neutral zones and overgrew into the area that was normally covered by the ablated cell (Fig. 6A, arrows). This overgrowth took place at 11 of 14 anterior borders and at 12 of 13 posterior borders of the operated hemisegments (Fig. 6B, $C$, and legend; Table 1). Two other cells that normally abut on the ablated ddaC also invaded the territory: $\mathrm{ddaC}$ in the contralateral hemisegment (Gao et al., 2000) (Table 1) and $v^{\prime}$ ada of the same class IV (data not shown).

The above result showed that $\mathrm{ddaC}$ is necessary for preventing its neighboring homologous cells from breaking the bounds of their dendritic fields. To address whether ddaC is sufficient for normal boundary formation of its neighbors, we performed a complementary ablation experiment using a pan-DA marker. In this experiment, all of the DA neurons in one dorsal cluster except for $\mathrm{ddaC}$ were ablated. As long as $\mathrm{ddaC}$ survived, we did not detect obvious in-growth ( 8 of 8 ablations at 16-17 hr AEL and 10 of 10 ablations at $25-30 \mathrm{hr}$ AEL) (Fig. 6D). This result corroborated the hypothesis that recognition specifically between adjacent ddaCs, but not between ddaC and cells of other classes, plays a pivotal role in preventing $\mathrm{ddaC}$ terminals from invading other areas. Dendritic fields of $\mathrm{ddaD}$ and $\mathrm{ddaE}$ did not overlap each other, but killing ddaD did not affect the field shape of ddaE in the same hemisegment or in an anterior neighbor (data not shown).

Loss of one of the two ddaC terminals, coming close to each other, triggered in-growth of the other one

In addition to the above results of the cell ablation experiments, we attempted to demonstrate the inhibitory interaction at the level of a single dendrodendritic interface. In larvae just after hatching (20-25 hr AEL), we looked for pairs of terminals of 


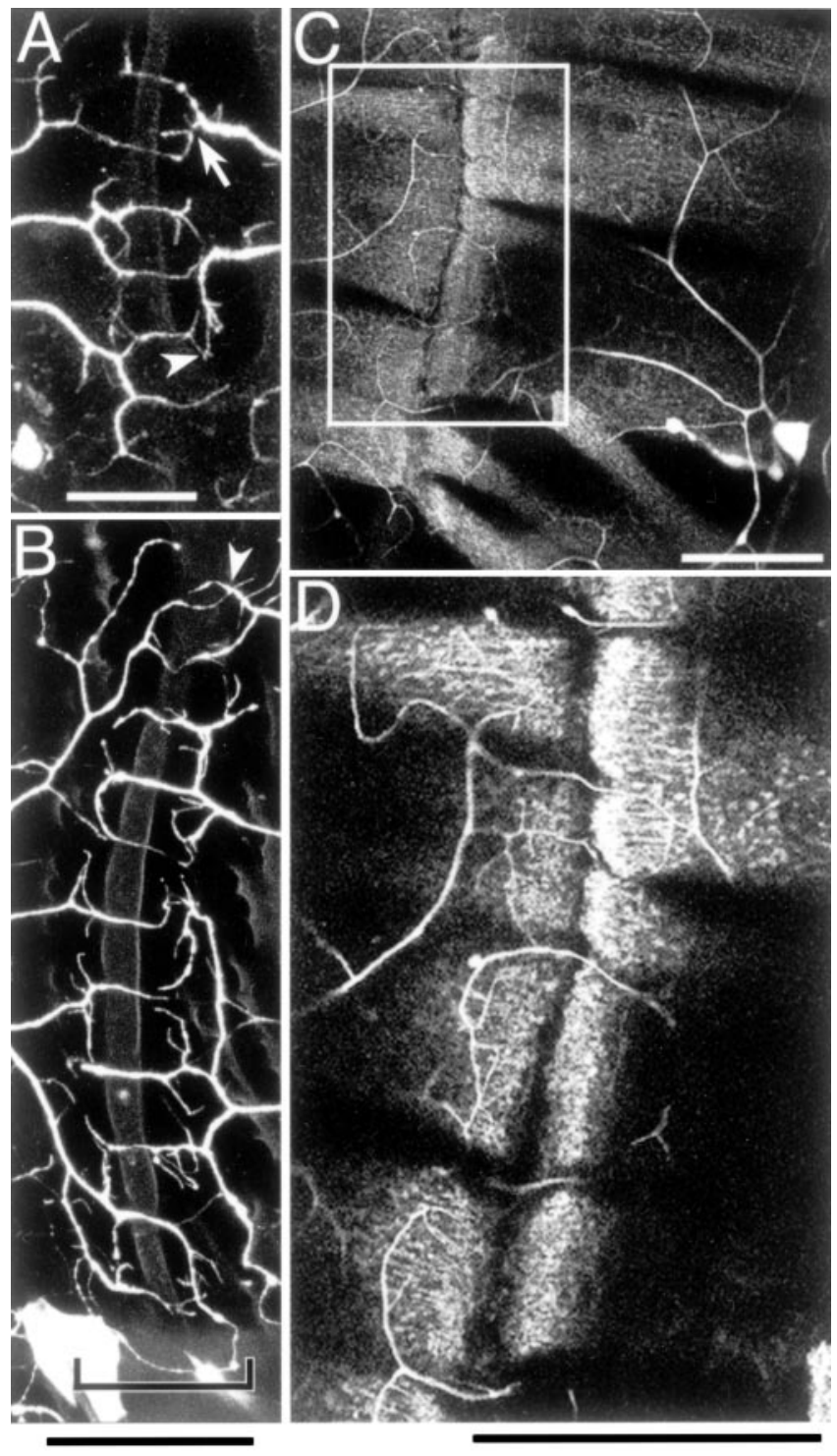

Figure 5. Observation of dendritic terminals of $\mathrm{ddaC}$ and coimaging with muscles. $A, B$ Images of two larvae of NP7028 GFP[S65T]/GFP[S65T] at 26-29 and 51-53 hr AEL, respectively. Close-up views of dendrodendritic contacts between adjacent $\mathrm{dda}$ C neurons. In the neutral zone (bracket in $B$ ), terminals were closely apposed or turned away before crossing each other (arrow) and sometimes it was difficult to identify dendritic tips (arrowheads). C, D, Venus-pm/+; NP7028 GFP[S65T]/MHC-CD8-GFP-Sh in a third instar larva. A muscle attachment site in C (box) was magnified in $D$. Dendrites passed through splits of muscle bundles ( $D)$. Scale bars: $A, 20$ $\mu \mathrm{m} ; B-D, 50 \mu \mathrm{m}$.

adjacent cells that came close to each other (Fig. $7 A-C$, green arrows). We then removed one terminal of the pair by cutting a proximal dendrite root and tracked how the other terminal behaved. The remaining terminals of either ipsilateral or contralateral homologs invaded territories that were normally covered by the detached branches (compare Fig. 7, compare $A, D, G ; B, E, H$; $C, F, I)$. This in-growth of heteroneuronal branches was seen in 12 of 14 cases. As observed in severing experiments of ddaC at 40 hr AEL, neighboring isoneuronal branches extended toward the severing site and grew into voided regions that were supposed to have been covered by the detached branches (Fig. 7, compare blue arrowheads in pairs of $A$ and $D, B$ and $E$ ). These isoneuronal branches looked as if they stood in the way of the in-growing heteroneuronal branches (Fig. $7 E$, blue arrowhead) or covered bounds of the neutral zones (Fig. $7 D, F$, purple arrowheads). All
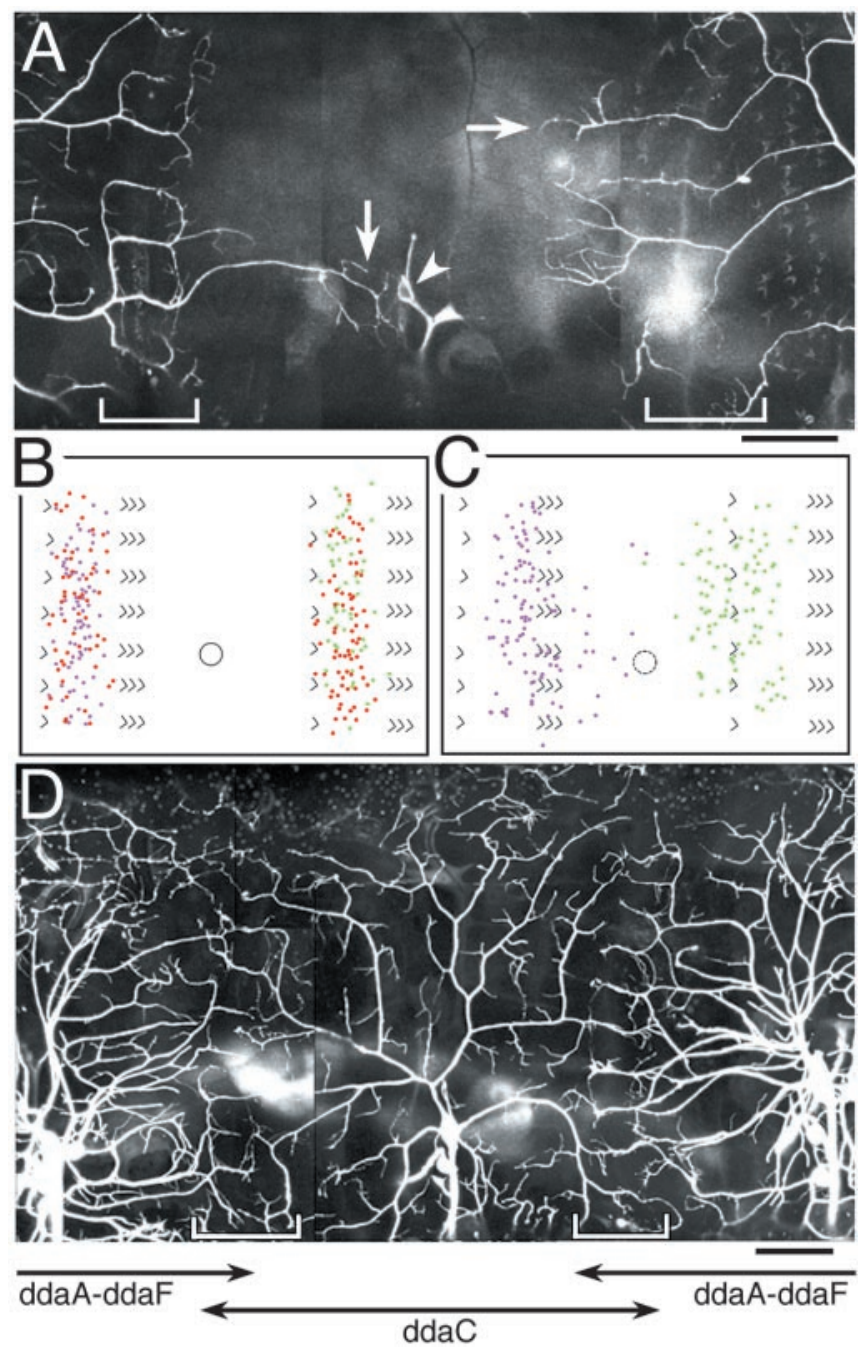

Figure 6. Ablation-induced ingrowth of adjacent $\mathrm{ddaC} . A$, $\mathrm{ddaC}$ was ablated at $20 \mathrm{hr}$ AEL in the embryo of NP7028 GFP[S65T]/GFP[S65T] and observed 2 later. Dendrites of adjacent ddaCs broke through the neutral zones (brackets) and invaded the area that was normally covered by the ablated homolog (arrows). Arrowhead points at an unknown cell. B, C, Positions of dendritic terminals of $\mathrm{dda} C$ were plotted in illustrations of the hemisegment. Each dot represents a single terminal. $B$, Positions of terminals in the control larva. Orange dots indicate dendritic ends of $\mathrm{ddaC}$ in the middle (circle). Violet dots and green dots indicate ends of an anterior adjacent $\mathrm{ddaC}$ and a posterior adjacent $\mathrm{ddaC}$, respectively. C, Positions of terminals of adjacent ddaCs when $\mathrm{ddaC}$ in the middle (circle with a broken line) was ablated. Violet dots on the left and green ones on the right are as described in $B$. D, All of the DA neurons in the dorsal cluster except for $\mathrm{ddaC}$ were ablated in embryos homozygous for 109(2)80 GFP[S65T] at 16-17 hr AEL, and observed 2 $\mathrm{d}$ later. In the middle hemisegment, only $\mathrm{ddaC}$ was left. Brackets indicate the neutral zones and dendrites of adjacent hemisegments did not transgress the borders. Scale bars: $A, D, 50 \mu \mathrm{m}$.

of these data support the hypothesis that inhibitory signaling is evoked between the growing tips when they encounter each other in development.

Sensitive periods for the lesion-induced in-growth of ddaC dendrites

During a series of severing experiments, we found that adjacent ddaCs often extended their dendrites beyond the neutral zone and entered the open area when branches were cut at 20-25 hr AEL (12 of 14 severings) but not at $40-43 \mathrm{hr}$ AEL (0 of 18) (compare Figs. $4 B, 7$ ). This comparison suggests that $\mathrm{ddaC}$ in the embryo and the young larva is able to respond to loss of its neighbor but that this dendritic plasticity is lost at subsequent larval 
Table 1. Sensitive periods for ablation-induced ingrowth of dendrites of adjacent DA neurons

\begin{tabular}{|c|c|c|c|c|c|}
\hline \multirow[b]{3}{*}{ Ablated cell(s) } & \multirow[b]{3}{*}{ Observed adjacent cell(s) } & \multicolumn{4}{|c|}{ Ingrowth into the field of the ablated cell(s) ${ }^{a}$} \\
\hline & & \multirow[b]{2}{*}{ Embryo } & \multicolumn{3}{|l|}{ Ablation at } \\
\hline & & & $25-30 \mathrm{hr}$ AEL & $35-40 \mathrm{hr}$ AEL & $45-50 \mathrm{hr}$ AEL \\
\hline $\mathrm{ddaC}$ & Anterior ddaC & $11 / 14$ & $3 / 13$ & $0 / 15$ & $0 / 14$ \\
\hline $\mathrm{ddaC}^{b}$ & Posterior ddaC & $12 / 13$ & $10 / 13$ & $0 / 12$ & $0 / 12$ \\
\hline $\mathrm{ddaC}$ and $\mathrm{ddaF}^{c}$ & Contralateral DA neurons & $9 / 10$ & n.d. & $2 / 14$ & n.d. \\
\hline
\end{tabular}

$\mathrm{ddaC}$ in one hemisegment was ablated at different stages, and ingrowth of dendrites from homologous neurons in either ipsilateral or contralateral hemisegments was quantified in terms of the number of hemisegments invaded. n.d., Not determined.

${ }^{a}$ As for arbors of either an anterior or posterior ipsilateral hemisegment, ingrowth was judged by whether terminals broke through the neutral zone and invaded into the field of the ablated cell (Fig. 6 A, $C$. Ingrowth into the ddaC field in a contralateral hemisegment was judged on the basis of territorial bounds between contralateral ddaCs in neighboring segments.

${ }^{b} \mathrm{ddaC}$ was ablated in $\sim 20 \mathrm{hr}$ AEL embryos or in larvae of NP7028 GFP[S65T]/GFP[S65T].

'Ablation of only ddaC caused ingrowth of the contralateral homolog, but to a limited extent; thus it was sometimes difficult to judge whether ingrowth occurred. Therefore both ddaC and ddaF were ablated in $16-17 \mathrm{hr}$ AEL embryos or in larvae of 109(2)80 GFP[S65T] homozygotes. This ablation protocol has been described previously (Gao et al., 2000).

stages. To examine this hypothesis, we ablated $\mathrm{ddaC}$ at different larval stages and recorded the positions of dendritic ends of adjacent ddaCs 2 d later. When the cell was ablated between 25 and $30 \mathrm{hr}$ AEL, dendrites of its ipsilateral segmental homolog overgrew into the voided region (Table 1). In contrast, ablation at 35-40 hr AEL or even later did not cause the transgression. Besides the reaction of ipsilateral $\mathrm{ddaC}$, in-growth of the contralateral DA neuron(s) was also much less dramatic and less penetrant when $\mathrm{ddaC}$ and ddaF were ablated after $35 \mathrm{hr}$ AEL (Table 1 , see details in the footnote). This stage sensitivity of ablation-induced in-growth was not restricted to ddaC; it was also the case with one class IV cell in the ventral cluster, vdaB, in terms of invasion by a contralateral homolog (data not shown).

\section{Discussion}

Recent studies have identified and characterized molecules that are important for regulating different aspects of pattern formation of dendrites (reviewed in Scott and Luo, 2001; Jan and Jan, 2001; Whitford et al., 2002). On the other hand, how distinct neuronal types generate considerable morphological heterogeneity of dendritic trees remains essentially unknown. To tackle this question, it would be ideal to monitor dendritic development of multiple cell types under study from its birth till maturation inside whole-mount animals. Our transgenic markers for da neurons are suitable for this purpose and allowed time-lapse observations of $\mathrm{ddaD}$ and ddaE (class I) and ddaC (class IV) at a single-cell resolution.

Distinct modes of dendritic branching The two classes of neurons used distinct strategies of dendritic emergence from the cell body and branching, which resulted in differences in their arbor patterns. ddaC increased in an arbor complexity mostly

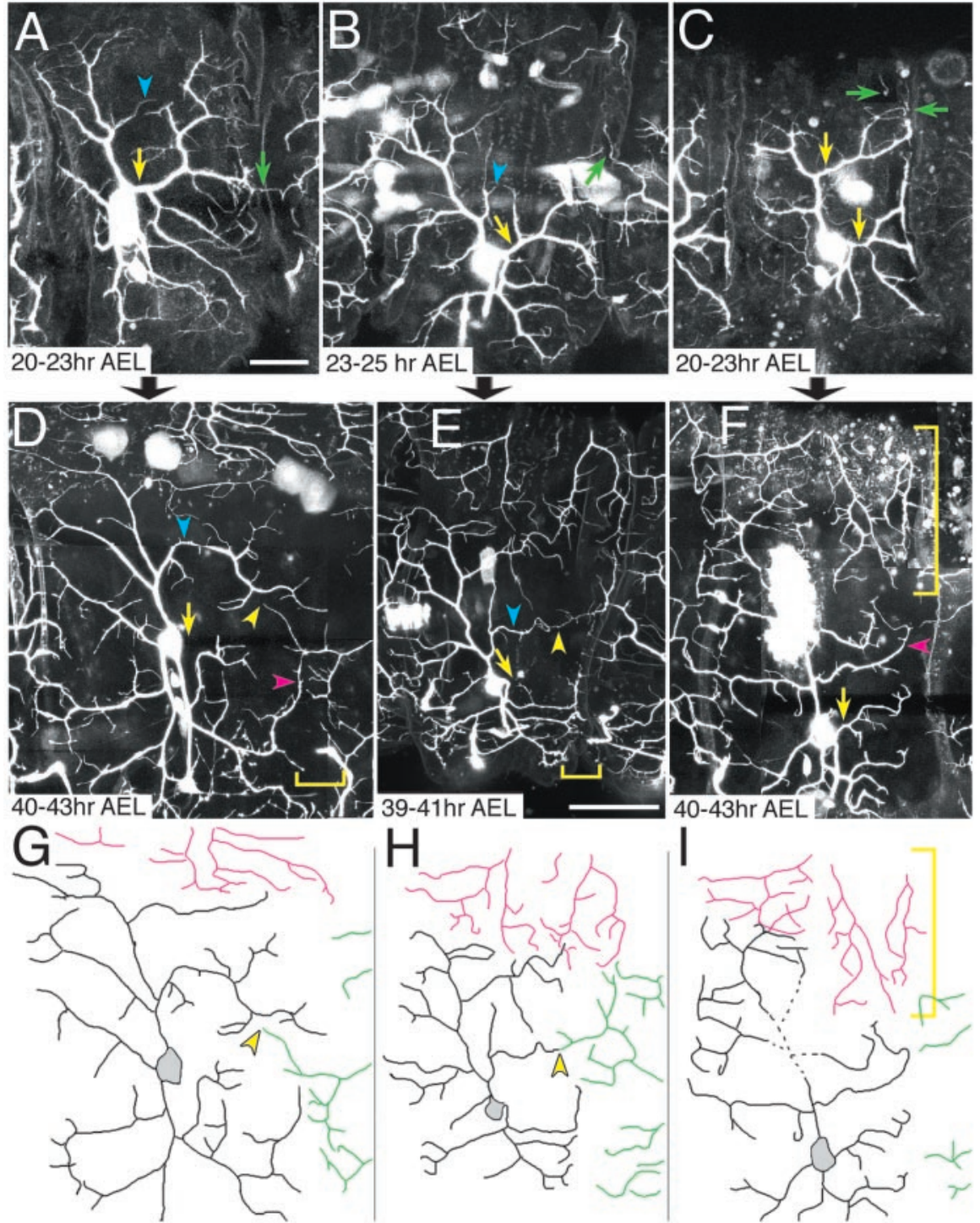

Figure 7. Loss of one of the two ddaC terminals, coming close to each other, triggered ingrowth of the other one. $A-C, D$ Dorsal clusters in larvae of NP7028 GFP[S65T]/GFP[S65T] just after hatching (20-25 hr AEL). Each green arrow indicates a dendrodendritic contact $(A)$ or a pair of terminals that were getting close to each other $(B, C)$. Yellow arrows represent proximal dendritic roots that were severed. $D-F$, Images of $16-20 \mathrm{hr}$ after severing of the roots as pointed in $A-C$, respectively. $G-I$, Tracings of dendrites of injured neurons (black), those of ipsilateral homologs (green), and those of contralateral ones (purple). The remaining terminals of either ipsilateral or contralateral homologs invaded territories that were normally covered by the detached branches (yellow arrowheads in $D, E, G$, and $H$ and brackets in $F$ and $I)$. Isoneuronal branches in $A$ and $B$ filled in voided fields as shown in $D$ and $E$, respectively (blue arrowheads), and some of them covered bounds of the neutral zones (purple arrowheads in $D$ and $F$ ). Brackets in $D$ and $E$ indicate the neutral zones. At 31-33 hr AEL between $B$ and $E$, we confirmed that the branch, which was targeted at 23-25 hr AEL (yellow arrow in $B$ ), was actually destroyed (data not shown). Scale bars: $A-C, 20 \mu \mathrm{m} ; D-F, 50 \mu \mathrm{m}$. 
by repeated splitting of dendritic ends in the embryo. At larval stages, $\mathrm{ddaC}$ formed lateral branches far behind the ends, which looked similar to the branching from the side of established shafts in ddaD and ddaE. Genetic screening for mutants was earlier performed by using a pan-DA marker, and several mutations were identified to affect overall branching patterns (Gao et al., 1999). Use of cell class-specific markers would facilitate the study of whether each of those loci is required for end splitting and/or lateral branch formation. Important goals would be to dissect the molecular basis of each of the two branching modes and to ultimately understand how they are differentially controlled within the same cell.

Putative mechanisms of filling-in reaction of ddaC dendrites In contrast with ddaD and ddaE that stabilized the shape of their entire arbors at early larval stages, ddaC continued to elaborate branches. Furthermore, ddaC exhibited a much greater capability of responding to open spaces. Severing of ddaC branches resulted in dramatic growth and sprouting from neighboring isoneuronal branches, and those late-emerging branches filled in voided regions, which is reminiscent of a study of cutting sensory arbors of a pressure-sensitive neuron in leech (Gan and Macagno, 1995). This filling-in response could be triggered by loss of local isoneuronal inhibitory contacts; alternatively, neighboring isoneuronal may have started competing for a putative growth factor that might be produced by potential target cells. Indifference of ddaD and ddaE to open areas could be explained by loss of reactions to such an extrinsic cue. Whichever mechanism elicits the filling-in reaction, the contact-meditated inhibition between isoneuronal branches prevents them from overlapping with each other.

The above and all of the other distinct natures of the two DA classes studied are probably linked to early genetic specification of each class. Thus, studies on genetic programming of dendritic pattern formation would be facilitated by identifying class selector genes and searching for their downstream targets. A search for molecules that are expressed in either one of the classes may isolate regulators that switch between class-specific features; such candidates may include $p d m 1$ and cut (Brewster et al., 2001; Grueber et al., 2002).

\section{A central role of inhibitory interaction between terminals of Class IV cells in tiling and possible roles of other cells}

Cell ablation studies using vertebrate retina support the probability of class-specific inhibitory interaction between heteroneuronal dendrites (Wässle et al., 1981; Perry and Linden, 1982; Weber et al., 1998). Due to limitations of cell labeling methods used, earlier studies could not perform time-lapse tracking of the same set of branch terminals. Therefore it was difficult to conclude whether terminal arbors of adjacent neurons initially overlap to a great extent followed by remodeling, or if they show mutual exclusion throughout development. Our recordings of heteroneuronal dendrites of ddaC support the latter possibility. Furthermore, our pinpoint surgery showed that ddaC-ddaC specific inhibitory interaction is necessary and sufficient for the dendritic exclusion. Finally, destroying a single dendro-dendritic interface showed that the inhibitory signaling occurs between the growing tips, through a contact-based mechanism or a short-range communication.

Although our result of the microsurgery highlights a crucial role of class-specific dendro-dendritic recognition in tiling, they do not necessarily exclude the contribution of surrounding cells to refining dendritic patterns. In response to ddaC ablation, all dendrite terminals of adjacent homologs, which were outgrowing within the neutral zone, did not necessarily break through. Ingrowth from anterior segments tended to be less penetrant than that from posterior ones. This could be explained by the hypothesis that DA neurons other than $\mathrm{ddaC}$, such as ddaB (Fig. 1F; Grueber et al., 2002), extend their dendrites toward the anterior segment boundary and those arbors may serve as physical barriers. Another example of surrounding tissues could be muscles, which may contribute to terminal extension with regular spacing.

\section{Interpretations of the sensitive period of the dendritic ingrowth}

Microsurgery-induced breaking of the bounds of ddaC was highly penetrant when lesions were made in the embryo; in contrast, the transgression was hardly detected when ddaC was ablated at 35-40 hr AEL or thereafter. This kind of the sensitive period for lesion-induced changes in dendritic morphology was reported also in the kitten retina (Eysel et al., 1985). Interestingly, our study of Drosophila DA neurons showed that this transition almost coincides with the stage when arbors of adjacent cells have come in touch with one another and the body wall has become almost completely tiled. This coincidence could be interpreted to indicate that the intercellular inhibitory signaling plays a pivotal role in establishing territorial bounds up to 35-40 hr AEL, but not in maintaining the bounds once they are defined.

\section{What is the underlying mechanism for ddaC-ddaC specific inhibitory interaction?}

Dendritic tiling has been studied well at the cellular level in the vertebrate retina and evidence has been obtained for cell classspecific recognitions, possibly through dendro-dendritic contacts (Lohmann and Wong, 2001). However, the underlying mechanisms remain to be elucidated. Thus we anticipate the future defining of the molecular mechanism of the inhibitory interaction specifically between class IV. It is proposed that flamingo, which encodes a seven-pass transmembrane cadherin (Usui et al., 1999), is required for the competitive interaction between dendrites of contralateral da neurons (Gao et al., 2000); on the other hand, dendrites of flamingo mutant ddaC mostly respect bounds between ipsilateral homologs (Grueber et al., 2002; K. S. and T. U., unpublished data). Phenotypes that are caused by the loss of flamingo function are further complicated, as was shown by the fact that $\mathrm{ddaD}$ and $\mathrm{ddaE}$ of class I overgrow their dendrites toward the dorsal midline (Sweeney et al., 2002; H. Kimura, T. Usui, and T. U., unpublished data).

How do dendrites of ddaC distinguish those of homologous cells from those of da neurons of other classes? The specific recognition between $\mathrm{ddaC}$ could be due to production by these neurons of a unique set of receptor and ligand to elicit the repulsive signaling. Alternatively, the cells could express a class-specific cell adhesion molecule that may recruit common repulsive signaling complexes to dendro-dendritic contact sites. In conclusion, we expect that further exploration into shaping of dendritic patterns of each class of da neurons will bring breakthroughs toward our understanding of the molecular basis for the diverse morphology of dendritic trees.

\section{References}

Becker S, Pasca G, Strumpf D, Min L, Volk T (1997) Reciprocal signaling between Drosophila epidermal muscle attachment cells and their corresponding muscles. Development 124:2615-2622.

Bodmer R, Jan YN (1987) Morpohological differentiation of the embryonic peripheral neurons in Drosophila. Roux's Arch Dev Biol 196:69-77.

Brand A (1995) GFP in Drosophila. Trends Genet 11:324-325.

Brand AH, Perrimon N (1993) Targeted gene expression as a means of al- 
tering cell fates and generating dominant phenotypes. Development 118:401-415.

Brewster R, Hardiman K, Deo M, Khan S, Bodmer R (2001) The selector gene $c u t$ represses a neural cell fate that is specified independently of the Achaete-Scute-Complex and atonal. Mech Dev 105:57-68.

Campos-Ortega JA, Hartenstein V (1997) . The embryonic development of Drosophila melanogaster. Berlin: Springer.

Devries SH, Baylor DA (1997) Mosaic arrangement of ganglion cell receptive fields in rabbit retina. J Neurophysiol 78:2048-2060.

Elston GN (2000) Pyramidal cells of the frontal lobe: all the more spinous to think with. J Neurosci 20:RC95(1-4).

Eysel UT, Peichl L, Wässle H (1985) Dendritic plasticity in the early postnatal feline retina: quantitative characteristics and sensitive period. J Comp Neurol 242:134-145.

Gan WB, Macagno ER (1995) Interactions between segmental homologs and between isoneuronal branches guide the formation of sensory terminal fields. J Neurosci 15:3243-3253.

Gao FB, Brenman JE, Jan LY, Jan YN (1999) Genes regulating dendritic outgrowth, branching, and routing in Drosophila. Genes Dev 13:2549-2561.

Gao FB, Kohwi M, Brenman JE, Jan LY, Jan YN (2000) Control of dendritic field formation in Drosophila: the roles of flamingo and competition between homologous neurons. Neuron 28:91-101.

Girdham CH, O'Farrell PH (1994) The use of photoactivatable reagents for the study of cell lineage in Drosophila embryogenesis. Methods Cell Biol 44:533-543.

Grueber WB, Graubard K, Truman JW (2001) Tiling of the body wall by multidendritic sensory neurons in Manduca sexta. J Comp Neurol 440:271-283.

Grueber WB, Jan LY, Jan YN (2002) Tiling of the Drosophila epidermis by multidendritic sensory neurons. Development 129:2867-2878.

Hayashi S, Ito K, Sado Y, Taniguchi M, Akimoto A, Takeuchi H, Aigaki T, Matsuzaki F, Nakagoshi H, Tanimura T, Ueda R, Uemura T, Yoshihara M, Goto S (2002) GETDB, a database compiling expression patterns and molecular locations of a collection of gal4 enhancer traps. Genesis 34:58-61.

Jan YN, Jan LY (1993) The peripheral nervous system. In: The development of Drosophila melanogaster (Bate M, Martinez-Arias A, eds), pp 12071244. Cold Spring Harbor, NY: Cold Spring Harbor Laboratory.

Jan YN, Jan LY (2001) Dendrites. Genes Dev 15:2627-2641.

Lee T, Luo L (1999) Mosaic analysis with a repressible cell marker for studies of gene function in neuronal morphogenesis. Neuron 22:451-461.

Lohmann C, Wong RO (2001) Cell-type specific dendritic contacts between retinal ganglion cells during development. J Neurobiol 48:150-162.
MacNeil MA, Masland RH (1998) Extreme diversity among amacrine cells: implications for function. Neuron 20:971-982.

Nagai T, Ibata K, Park ES, Kubota M, Mikoshiba K, Miyawaki A (2002) A variant of yellow fluorescent protein with fast and efficient maturation for cell-biological applications. Nat Biotechnol 20:87-90.

Oda H, Tsukita S (1999) Dynamic features of adherens junctions during Drosophila embryonic epithelial morphogenesis revealed by a Dalphacatenin-GFP fusion protein. Dev Genes Evol 209:218-225.

Perry VH, Linden R (1982) Evidence for dendritic competition in the developing retina. Nature 297:683-685.

Ramón y Cajal S (1995) Histology of the nervous system of man and vertebrates. Oxford: Oxford UP.

Sawano A, Hama H, Saito N, Miyawaki A (2002) Multicolor imaging of $\mathrm{Ca}(2+)$ and protein kinase $\mathrm{C}$ signals using novel epifluorescence microscopy. Biophys J 82:1076-1085.

Schüpbach T, Wieschaus E (1998) Probing for gene specificity in epithelial development. Int J Dev Biol 42:249-255.

Scott EK, Luo L (2001) How do dendrites take their shape? Nat Neurosci 4:359-365.

Shiga Y, Matakatsu M, Hayashi S (1996) A nuclear GFP/beta-galactosidase fusion protein as a marker for morphogenesis in living Drosophila. Dev Growth Differ 38:99-106.

Sweeney NT, Li W, Gao FB (2002) Genetic manipulation of single neurons in vivo reveals specific roles of flamingo in neuronal morphogenesis. Dev Biol 247:76-88.

Usui T, Shima Y, Shimada Y, Hirano S, Burgess RW, Schwarz TL, Takeichi M, Uemura T (1999) Flamingo, a seven-pass transmembrane cadherin, regulates planar cell polarity under the control of Frizzled. Cell 98:585-595.

Verkhusha VV, Tsukita S, Oda H (1999) Actin dynamics in lamellipodia of migrating border cells in the Drosophila ovary revealed by a GFP-actin fusion protein. FEBS Lett 445:395-401.

Wässle H, Peichl L, Boycott BB (1981) Dendritic territories of cat retinal ganglion cells. Nature 292:344-345.

Weber AJ, Kalil RE, Stanford LR (1998) Dendritic field development of retinal ganglion cells in the cat following neonatal damage to visual cortex: evidence for cell class specific interactions. J Comp Neurol 390:470 -480.

Whitford KL, Dijkhuizen P, Polleux F, Ghosh A (2002) Molecular control of cortical dendrite development. Annu Rev Neurosci 25:127-149.

Zito K, Parnas D, Fetter RD, Isacoff EY, Goodman CS (1999) Watching a synapse grow: noninvasive confocal imaging of synaptic growth in Drosophila. Neuron 22:719-729. 